\title{
Optical Frequency Comb Generation Using Dual-Mode Injection-Locking of Quantum-Dash Mode-Locked Lasers: Properties and Applications
}

\author{
Ehsan Sooudi, Student Member, IEEE, Stylianos Sygletos, Andrew D. Ellis, Guillaume Huyet, \\ Senior Member, IEEE, John G. McInerney, Senior Member, IEEE, François Lelarge, Kamel Merghem, Ricardo \\ Rosales, Anthony Martinez, Abderrahim Ramdane, and Stephen P. Hegarty, Member, IEEE
}

\begin{abstract}
In this paper, we describe generation and application of wide, narrow linewidth optical frequency combs using dualmode injection-locking of InP quantum-dash mode-locked lasers. In the first part, the dependence of the RF locking-range on the device's absorber voltage is experimentally investigated. Under optimized absorber voltage, a continuous, wide RF locking-range of $\approx 400 \mathrm{MHz}$ is achievable for lasers with $21 \mathrm{GHz}$ repetition rate. The total RF locking-range of $\approx 440 \mathrm{MHz}$ is possible considering of locking-range for positive and negative absorber voltages. This wide tuning $>2 \%$ of the repetition rate, a record for a monolithic mode-locked laser, is reported from a two-section device without any additional passive section or extended-cavity for repetition rate tuning. It is shown that the effective $R F$ locking-range in dual-mode injection corresponds to the optical locking-range and repetition rate tuning under $\mathrm{CW}$ injection which is wider when the free-running mode-locking operation is "less stable". The widest comb consists of 35 narrow lines within $10 \mathrm{~dB}$ of the peak, spanning $\approx 0.7 \mathrm{THz}$, generating $3.7 \mathrm{ps}$ pulses. In the second part, we show the first demonstration of multi pump phase-synchronization of two $10 \mathrm{~Gb} / \mathrm{s}$ DPSK channels in a phasesensitive amplifier using dual-mode injection-locking technique. The phase-sensitive amplifier based on "black box" scheme shows more than $7 \mathrm{~dB}$ phase-sensitive gain and error free performance for both input channels with $1 \mathrm{~dB}$ penalty.
\end{abstract}

Index Terms-Mode-locked lasers, optical-injection-locking, optical frequency combs, quantum-dash lasers, and phasesensitive amplifiers.

\section{INTRODUCTION}

$\mathbf{C}$ OHERENT optical frequency combs (OFCs) with narrow optical linewidths have various applications in optical

Manuscript received 05 April, 2012; revised 21 June 2012. This work was conducted under the framework of the INSPIRE programme, funded by the Irish Government's PRTLI Cycle 4, National Development Plan 2007-2013, and also supported by the Science Foundation Ireland (SFI) under Contract 07/IN.1/I929 and European Communities Seventh Framework Programme FP/2007-2013 under grant 224547 (PHASORS). This work was also funded by the EU FP7 Marie Curie Action FP7-PEOPLE-2010-ITN through the PROPHET project, Grant No. 264687.

E. Sooudi, S. Sygletos, A. D. Ellis, and J. G. McInerney are with the Department of Physics, University College Cork, Cork, Ireland, and Tyndall National Institute, Lee Maltings, Cork, Ireland (e-mail: Ehsan.Sooudi@tyndall.ie).

G. Huyet and S. P. Hegarty are with CAPPA, Cork Institute of Technology, Cork, Ireland and Tyndall National Institute, Lee Maltings, Cork, Ireland.

F. Lelarge is with the III-V Lab, a joint Laboratory of "Alcatel Lucent Bell Labs", "Thales Research \& Technology" and "CEA-LETI", Route de Nozay, 91460, Marcoussis, France.

K. Merghem, R. Rosales, A. Martinez, and A. Ramdane are with the Laboratory for Photonics and Nanostructures, CNRS, Marcoussis 91460, France. communication systems such as multi-carrier transmission systems in orthogonal frequency division multiplexing (OFDM) [1], coherent wavelength division multiplexing (CoWDM) [2], arbitrary waveform generation [3], [4], all optical signal processing [5], and millimeter-wave generation [6]. A number of techniques have been proposed for the generation of OFCs such as using cascaded intensity and/or phase modulators [7], [8], fibre or semiconductor based ring-cavity mode-locked lasers [9], [10], direct modulation of gain-switched discretemode lasers [11], and semiconductor mode-locked lasers [12].

Despite the fact that OFCs from semiconductor modelocked lasers have inherently wide spectral width comparing the other OFC generation techniques, several performance limiting factors reduce their suitability for this purpose. First, the timing jitter of passively mode-locked lasers (an indication of coherence among comb lines) is not usually adequate, so an stabilization technique is required. Hybrid/active mode-locking could be used to synchronize the repetition rate of the laser to that of the external RF source. However, the RF lockingrange is usually limited to few tens of $\mathrm{MHz}$ unless a specially designed RF circuit is utilized along with a complex multisection cavity structure for wide tuning of the repetition rate [13]-[15]. Such developments have produced locking-ranges of $500 \mathrm{MHz}$ for hybrid [14] and $1.8 \mathrm{GHz}$ for actively modelocked lasers at $40 \mathrm{GHz}$ [15]. Increasing the RF locking-range, beyond the cleaving error of the device, is highly desirable to compensate any process related error between the repetition rate of the device and the pre-defined frequency of the system. This makes the use of mode-locked laser based OFC suitable for applications based on pre-defined clock frequency.

The second limiting factor is that the optical linewidth of individual lines of a mode-locked laser can be large $(\sim 10-100$ $\mathrm{MHz}$ ) while sub $\mathrm{MHz}$ to few $\mathrm{MHz}$ is desirable in applications such as phase-encoded modulation formats. It is important to note that neither hybrid nor active mode-locking techniques influence the individual line's frequency noise. Therefore, a technique leading to noise reduction of the mutual and individual OFC lines from mode-locked laser is highly desirable to generate high quality OFCs with narrow optical linewidths and small RF beating noise.

Injection-locking of two coherent $\mathrm{CW}$ lines (dual-mode injection) can be used to synchronize two independent OFCs from mode-locked lasers [16]. Carrier modulation due to beating of the two injected lines gives control over the 
repetition rate and timing jitter of the slave laser unlike $\mathrm{CW}$ injection-locking of a mode-locked laser. This scenario could be simplified by generating the dual-modes from an amplitude modulated CW line rather than a stabilized modelocked laser in [16]. A narrow-linewidth CW source generates two sidebands with narrow optical linewidth after amplitude modulation, which are then used for injection-locking of a mode-locked laser to generate OFCs with narrow optical linewidth and low RF beating noise. This is an important advantage over hybrid/active mode-locking techniques.

In this paper, we demonstrate generation of narrow linewidth OFCs with wide RF locking-range and few ps pulse-width using dual-mode injection of two-section $21 \mathrm{GHz}$ quantum-dash mode-locked lasers (QDMLs). It is shown experimentally that the RF locking-range under dual-mode injection is correlated with the repetition rate tuning and optical locking-range under $\mathrm{CW}$ injection-locking. Less stable mode-locked operation at free-running leads to wider optical locking-range and repetition rate tuning under $\mathrm{CW}$ injection and greater RF locking-range under dual-mode injection. A wide RF locking-range of $400 \mathrm{MHz}$ is reported for fixed bias parameters by adjusting the master laser's wavelength and frequency of driving RF source within the locking-range. This brings the total possible RF locking-range to more than $440 \mathrm{MHz}$ by varying the bias parameters without any passive section for extra tuning of the repetition rate. To the best of our knowledge, this is the highest tuning ratio $(\approx 2.1 \%)$ obtained from a monolithic mode-locked laser. In the second part of the paper, we introduce a novel application of OFCs generated with this technique in multi-channel phase-sensitive amplifiers (PSAs). For the first time, two lines of the OFC are utilized as local pump source for a PSA having two coherent $10.374 \mathrm{~Gb} / \mathrm{s}$ WDM channels at $41.496 \mathrm{GHz}$ spacing as the input. Using this technique the phase synchronization of pumps for both channels is derived from a single injection-locking stage. The dual-modes were two coherent carriers generated through fourwave mixing (FWM) of a local pump and two DPSK channels, forming the carrier extraction stage of the "black box" PSA. More than $7 \mathrm{~dB}$ gain swing and error free performance for both channels with $1 \mathrm{~dB}$ penalty is demonstrated at the output of the PSA.

\section{EXPERIMENTAL ARRANGEMENT FOR INJECTION LOCKING AND DEVICE DESCRIPTION}

The experimental arrangement for injection-locking was a master-slave configuration where only the light from the master was unidirectionally injected to the slave laser. As shown in Fig. 1, light from the master source passed through a variable optical attenuator, a coupler (with a tap for monitoring the injected power), polarization controller and entered Port 1 of a circulator. The light from Port 2 of the circulator was fed into the facet of the gain-section of the slave laser using a lensed fiber. The light from the facet of the saturableabsorber section was utilized for optical/RF spectrum analysis through free-space coupling with an isolator. For the RF spectrum analysis, the light passed though a high speed photodetector (Newport, Model: D-25xr) with bandwidth of $\approx 17$

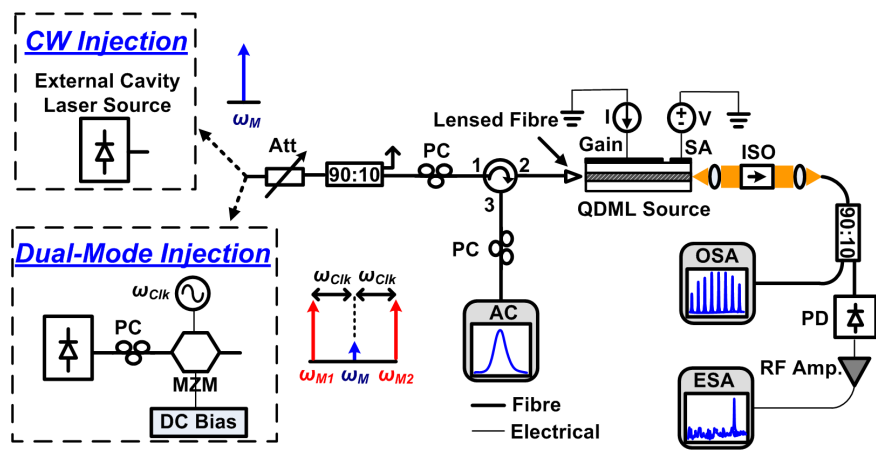

Fig. 1. The schematic of experimental arrangement used for CW and Dualmode injection-locking. The definition of abbreviations used: PC: Polarization controller, MZM: Mach-Zehnder Modulator, Att. Variable Optical Attenuator, PD: Photodetector, SA: Saturable-Absorber, ISO: Free space isolator, OSA: Optical Spectrum Analyzer, ESA: Electrical Spectrum Analyzer, AC: Autocorrelator, Amp.: Amplifier, QDML: Quantum Dash Mode-Locked Laser.

$\mathrm{GHz}$ and RF amplifier (0.1-18 GHz). The Port 3 output of the circulator was then used for measurement of the pulsewidth using a background-free second harmonic generation autocorrelator. The master laser was a commercial single-mode external-cavity tunable laser (NewFocus, model: 6328) with optical linewidth $<1 \mathrm{MHz}$. As depicted in Fig. 1, two cases were considered for the analysis of optical injection. Case 1 was $\mathrm{CW}$ injection, where the master laser's $\mathrm{CW}$ output was used for optical injection. Case 2 was dual-mode injection, where the light from the master laser passed through a MachZehnder modulator driven by an RF source and DC biased to generate two coherent $\mathrm{CW}$ side-bands and suppress the original master laser mode. The spacing of the side-bands was twice the frequency of the RF source as shown in Fig. 1. The optical linewidth of the longitudinal modes of the slave laser was measured using heterodyne detection with another narrow linewidth tunable laser source.

The two InP QDMLs used were both two-section devices with an active layer comprising 9 InAs dash monolayers grown by gas source MBE embedded within two barriers and separate confinement heterostructure (SCH) layers (dash in a barrier structure). Both the barriers and $\mathrm{SCH}$ layers consisted of $\mathrm{In}_{0.8} \mathrm{Ga}_{0.2} \mathrm{As}_{0.4} \mathrm{P}_{0.6}$ quaternary materials with $\lambda_{g}=1.17 \mu \mathrm{m}$ [17]. Total cavity length of each device was $2030 \mu \mathrm{m}$ with absorber lengths of $140 \mu \mathrm{m}$ (length ratio $\approx 6.9 \%$ ) and $190 \mu \mathrm{m}$ (length ratio $\approx 9.4 \%$ ). The absorber and gain sections were isolated by a resistance $>10 \mathrm{k} \Omega$. The QDMLs were mounted p-side up on AlN submounts and copper blocks with active temperature control and electrical contacts formed by wirebonding. From now on, we call the laser with longer absorber " $D_{1}$ " and the device with shorter absorber as " $D_{2}$ ". Both devices operated in saturable-absorber dominated modelocked (SAML) regimes [18], [19] when the absorbers were biased well below the transparency point $(\approx 0.9 \mathrm{~V})$. The bias operational parameters of the device for SAML operation were typically around 100-200 mA for gain current and approximate absorber voltage of $-0.6 \mathrm{~V}$ to $0.3 \mathrm{~V}$ for $D_{1}$ and $-1.1 \mathrm{~V}$ to $0.3 \mathrm{~V}$ for $D_{2}$. No lasing was observed for absorber voltages biased below these values. Both the devices showed averaged freespace output powers of a few $\mathrm{mW}$ in this regime of operation. 
By optimizing the bias parameters (absorber bias around 0.1$0.2 \mathrm{~V}$ and gain current around $50 \%$ above threshold) relatively narrow RF linewidth (10s-100s of $\mathrm{kHz}$ ) and pulses of a few ps in duration were produced in free-running operation.

Optical injection improved the lasers' performance in several aspects. In the optical domain, the spectral width of the laser was reduced with similar or narrower pulses, thereby reducing the time-bandwidth product of the pulses. In the RF domain, we observed a shift in the RF peak to higher frequencies, and a reduction in RF linewidth (at relatively low injection powers) using $\mathrm{CW}$ injection. Full control over the RF linewidth of the device was possible using dualmode injection which strongly narrowed the RF linewidth, similar to hybrid mode-locking. We analyze the dependence of the optical and RF locking-range of the injection-locked device on the bias parameters. It will be shown that the locking characteristics, particularly the RF locking-range could be significantly improved by careful adjustment of the bias parameters. For this purpose, the devices were biased under two different gain current and absorber voltages to show "distinct" SAML operation in terms of RF linewidth and pulsewidth. The optical spectra of $D_{1}$ and $D_{2}$ for two different bias parameters are shown in Fig. 2(a) and (b), respectively. As can be seen, decreasing the absorber bias to the corner of the SAML operating range $\left(-0.5 \mathrm{~V}\right.$ for $D_{1}$ and $-1.0 \mathrm{~V}$ for $\left.D_{2}\right)$ caused narrowing the optical spectrum. The spectral narrowing was accompanied by a decrease in the output power and an increase of threshold current for the devices. The gain current for the negative absorber case was increased so that the average power of the devices remained the same as that of the positive absorber voltage.

The intensity autocorrelation of the devices are shown in Fig. 3(a) and (b). The traces were intentionally offset for better viewing. The pulse-width after deconvolution for $D_{1}$ was 7.2 ps (Gaussian fit) reducing to $4.0 \mathrm{ps}\left(\mathrm{Sech}^{2}\right.$ fit). For $D_{2}$ the pulsewidths were 5.6 ps (Gaussian fit) reducing to $3.3 \mathrm{ps}$ ( $\mathrm{Sech}^{2}$ fit) when the absorber is negatively biased. For both cases a clear coherence spike could be seen in the traces for negative absorber voltages. It is unlikely that these come from partial dispersion compensation in the fibre as only a few meters of single-mode fibre was used in the signal path to the autocorrelator. The existence of such peaks could indicate instabilities or some excess noise in the mode-locking operation. The RF linewidth of the devices at these two regions also showed significant differences. For $D_{1}$ the RF linewidth at positive absorber voltage was around few $100 \mathrm{~s}$ of $\mathrm{kHz}$ which was increased to few $\mathrm{MHz}$ for negative absorber voltage. The same trend was observed for $D_{2}$ where the RF linewidth of few $10 \mathrm{~s}$ of $\mathrm{kHz}$ at positive absorber voltage was increased to a few $100 \mathrm{~s}$ of $\mathrm{kHz}$ when the absorber was negatively biased. Information on RF linewidth and autocorrelation traces could be an indication of "less stable" mode-locking operation for absorber biased close to the negative boundary of modelocking operation. From hereon, the measurement results on $D_{1}$ will be presented which were qualitatively similar to those of $D_{2}$.

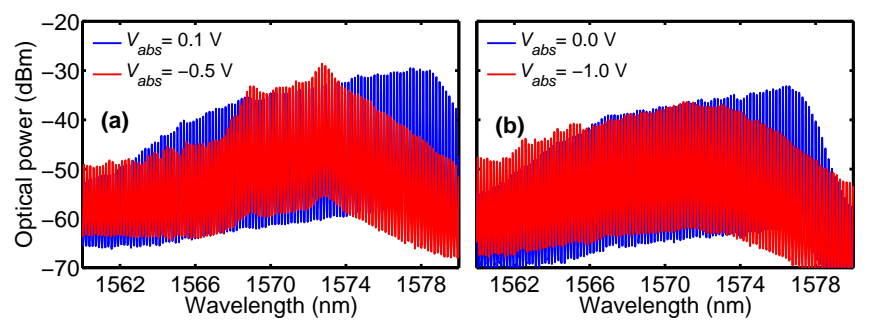

Fig. 2. The optical spectrum of two-section QDMLs at free running operation. (a) $D_{1}$, Gain current: $160 \mathrm{~mA}$, Absorber voltage: $0.1 \mathrm{~V}$ and Gain current: $200 \mathrm{~mA}$, Absorber voltage: $-0.5 \mathrm{~V}$. (b) $D_{2}$, Gain current: $110 \mathrm{~mA}$, Absorber voltage: $0.0 \mathrm{~V}$ and Gain current: $135 \mathrm{~mA}$, Absorber voltage: -1.0 V.
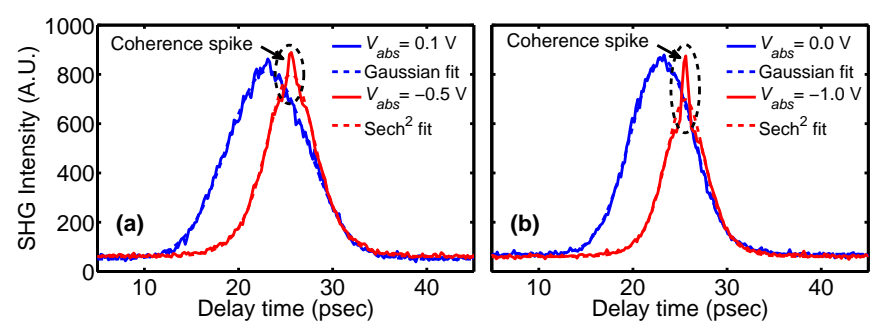

Fig. 3. Autocorrelation measurement of two-section QDMLs at free running operation. (a) $D_{1}$ corresponding to Fig. 2(a). (b) $D_{2}$ corresponding to Fig. 2(b).

\section{ANALYSIS OF REPETITION RATE TUNING}

Repetition rate tuning is an important parameter to assess the suitability of mode-locked semiconductor lasers for a real application where some frequency deviation exists between laser's repetition rate (given by its optical length) and that of the system. In this section, we analyze the amount of repetition rate tuning in the free running and $\mathrm{CW}$-injection cases.

\section{A. Free running}

Fig. 4 shows the amount of frequency change for $D_{1}$ in two cases: (a) when the absorber voltage and temperature of the mount are fixed and only gain current is changed, (b) where both the bias parameters are fixed and temperature of mount is changed. Several differences can be distinguished from Fig. 4(a): first, an increase in the repetition rate has been observed when the absorber voltage was decreased from positive to negative values. Second, the trend of tuning against the gain current for positive absorber is descending while it is ascending for negative absorber voltages. Third, the amount of tuning is around $30 \mathrm{MHz}$ for positive absorber voltage while it increases up to $100 \mathrm{MHz}$ when the absorber is negatively biased. The same kind of trend was observed when the bias parameters are fixed and only the temperature of the mount is changed. The trend for positive absorber voltage was ascending for positive absorber bias in the range of $\approx 3 \mathrm{MHz}$ (not visible in Fig. 4(b)). When the temperature was changed with a negative absorber bias, the trend of frequency tuning was descending and large amount of tuning $(\approx 100 \mathrm{MHz})$ could be seen.

The mechanism for the difference in the trend and the amount of frequency tuning for the two cases seems not to be 


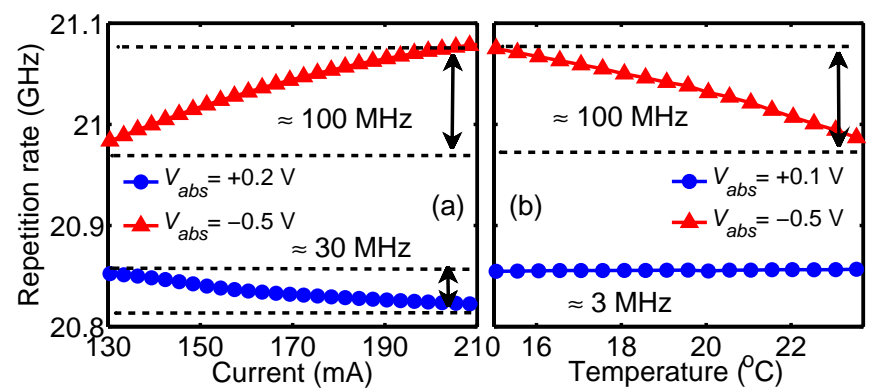

Fig. 4. Free-running repetition rate of $D_{1}$ for different bias parameters. (a) Absorber voltages: $0.2 \mathrm{~V}$ and $-0.5 \mathrm{~V}$, mount temperature: $20.0^{\circ} \mathrm{C}$, gain current ranging from 130 to $210 \mathrm{~mA}$. (b) Absorber voltages: $0.1 \mathrm{~V}$ and -0.5 $\mathrm{V}$, gain current: $160 \mathrm{~mA}$ and mount temperature $15-23.5^{\circ} \mathrm{C}$.

only a function of change in the refractive index. For example, if we consider plasma effect (change of refractive index due to change in the carrier density) the repetition rate should always increase when the gain current is increased. However, we observed both ascending and descending trends for different absorber voltages. Another explanation could be presented using the definition of the "detuning time" (the actual arrival time of the pulse according to the gain/absorption saturation time). This will cause the pulse repetition rate to deviate from the Fabry-Perot fundamental frequency [20]. It was shown that the amount of detuning time is basically a function of pulse energy, the pulsewidth and detailed saturation dynamics which are governed by gain/absorber bias parameters [21]. Therefore, it is possible that the laser could be biased in a region so that the detuning time will be an ascending or descending function of the gain current and also with more or less sensitivity.

\section{B. CW optical injection}

Following study of repetition rate detuning in free running mode-locked operation, we analyzed the $\mathrm{CW}$ injection-locking behavior of the laser at the same bias parameters as shown in Fig. 2. Here, stable locking is defined as the regime where the laser operation meets the following criteria. First, in the RF domain, no beating frequency or oscillations associated with relaxation oscillation (RO) frequency (instabilities under optical injection) existed at low frequency range (0- few $\mathrm{GHz}$ ). By zooming around the repetition rate, the shift of the repetition rate to a new (larger) frequency was observed. More than $20 \mathrm{~dB}$ suppression of laser's original RF spectral peak was also considered to determine the unlocking-locking transition. For positive values of the absorber voltage, there was an abrupt frequency shift by $>150 \mathrm{MHz}$ whereas at negative bias gradual shift by $\sim 10 \mathrm{MHz}$ for negative voltages was observed. Second, in the intensity autocorrelation trace, the coherence spike observed when free running with negative absorber bias disappeared. This is a likely indication of suppression of free-running instabilities in pulses generated by the injectionlocked mode-locked laser. Third, in the optical spectrum of the injection-locked laser, spectral narrowing occurred in the vicinity of injection with significant portions of the spectral power in modes shifted to longer wavelengths by a few nanometers [18].

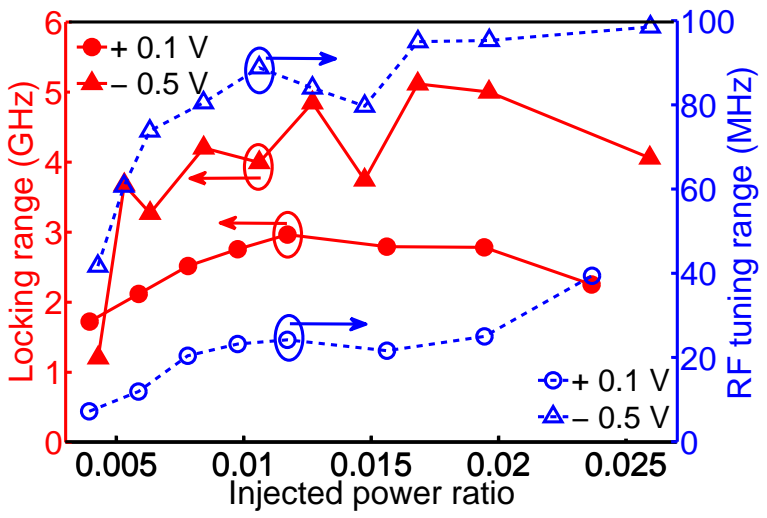

Fig. 5. Optical locking-range (left axis) at positive absorber voltage (full circles) and negative absorber voltage (full triangles) and repetition rate tuning (right axis) at positive absorber voltage (empty circles), and negative absorber voltage (empty triangles) versus injected power ratio. The bias parameters are the same as in Fig 2(a).

In Fig. 5, the optical locking-range (filled markers) and the amount of repetition rate tuning (empty markers) within the locking-range for two absorber voltages are shown in the left and right axes, respectively. The injection ratio (" $x$ " axis) is defined as the ratio of injected power to the free running slave's power. As can be seen, the locking-range for the negative absorber voltage was significantly wider than for the positive absorber voltage. For this device, a locking-range up to 5.1 $\mathrm{GHz}$ was found which is a large fraction of the longitudinal mode-spacing $(\approx 21 \mathrm{GHz}$ ). This widening of the locking-range was predicted theoretically in a model for GaAs quantumdot mode-locked lasers around the boundaries of operational map for passive mode-locking, where "unstable" mode-locking evolves in free running operation [22]. In our case, the wider optical locking-range corresponded to an absorber voltage of $-0.5 \mathrm{~V}$ while the negative boundary of passive mode-locking operation was $-0.6 \mathrm{~V}$. In addition to the wide optical lockingrange, the amount of repetition rate tuning within the lockingrange was considerably different in the two cases. The maximum amount of repetition rate tuning for the positive absorber voltage (empty circles, right axis) is around $40 \mathrm{MHz}$ while it increases to $\approx 100 \mathrm{MHz}$ for the negative absorber voltage (empty triangles, right axis). This difference might arise from the initial bias conditions for the gain and absorber sections that provided a significant repetition rate tuning difference in free-running operation. However, the contribution of injectionlocking in changing the detuning time and refractive index when the master laser's frequency is swept across the lockingrange is presently unclear and requires a separate investigation which is not within the scope of this paper. Fig. 6 shows the change in the repetition rate within the locking-range for positive (filled circles) and negative (filled triangles) absorber voltages when the master laser is swept. The point where the stable locking emerges was considered as the reference for the master frequency ( 0 value). The injected power ratio was almost the same (1.1\% for positive and $1.2 \%$ for negative absorber voltage) for the two cases. The free running repetition rate for the positive absorber bias was $\approx 20.858 \mathrm{GHz}$ which was up-shifted by $\approx 168 \mathrm{MHz}$ under stable injection-locking. 


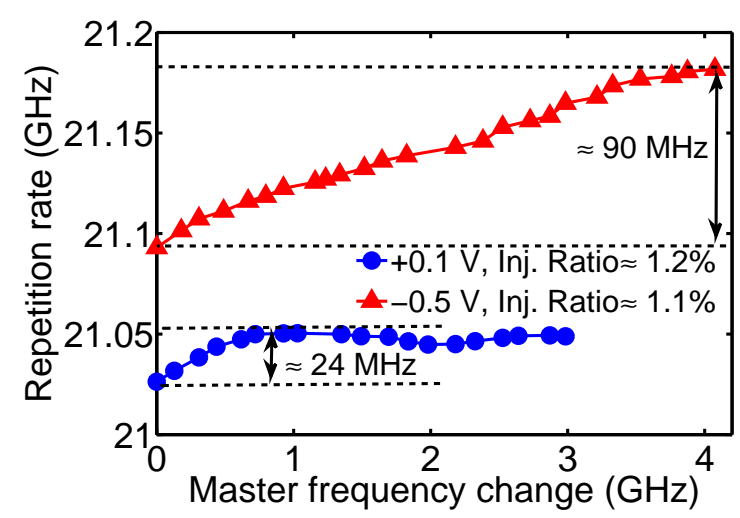

Fig. 6. Repetition rate within the locking-range as a function of master laser frequency change for positive absorber voltage (circles, injected power ratio $\approx 1.1 \%$ ) and negative absorber voltage (triangles, injected power ratio $\approx 1.2 \%$ ). Bias parameters were the same as Fig. 5 .

For the negative absorber voltage, the free running repetition rate was $\approx 21.026 \mathrm{GHz}$ (already shifted to higher frequencies than that of positive absorber voltage) was up-shifted by $\approx 66$ $\mathrm{MHz}$ at the evolution of stable locking. A noticeable difference could be seen for the trend of repetition rate tuning for both cases: the repetition rate monotonically increased within the locking-range for negative absorber bias. However, for the positive absorber bias, a non-monotonic trend (first ascending followed by a descending trend and some "clamping" of the maximum frequency) could be observed within the lockingrange. This kind of trend was observed for other injection levels depicted in Fig. 5. Total repetition rate tuning of $\approx 24$ $\mathrm{MHz}$ was achieved for positive absorber voltage while it increased to $\approx 90 \mathrm{MHz}$ for the negative absorber voltage.

As will be shown later, the combination of wide lockingrange and large repetition rate tuning range under $\mathrm{CW}$ injection-locking leads to much larger RF locking-range under dual-mode injection.

\section{VERSATILE OPTICAL FREQUENCY COMB GENERATION USING DUAL-MODE OPTICAL INJECTION}

In this section, we describe dual-mode injection-locking of $D_{1}$. Locking characteristics of $\mathrm{CW}$ injection showed that the lasers operating at negative absorber bias have wider optical locking-range and tuning of repetition rate within the locking-range. Injection of two coherent $\mathrm{CW}$ lines (dual-mode injection), bringing the two lines close enough (in wavelength) to two slave laser modes, causes modulation of the carriers due to saturation of the gain. Careful adjustment of this modulation frequency results in RF frequency locking, as well as enhanced optical injection-locking. The modulation frequency is the spacing of the two lines, or a sub-harmonic where dual-mode injection is done at an integer harmonic of the repetition rate. The spacing of the two coherent modes should be consistent with the shift of the repetition rate associated with the injection-locking process (for example, see Fig. 6).
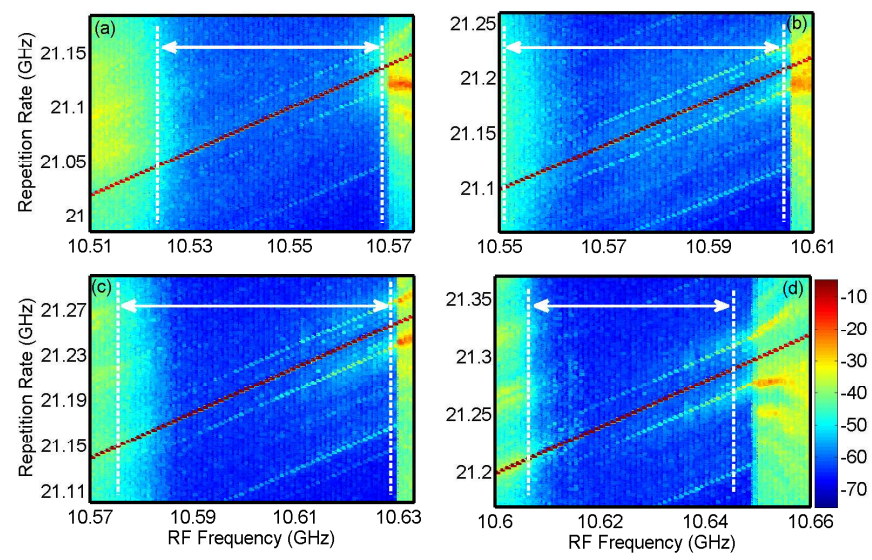

Fig. 7. Intensity graphs for the RF spectrum under dual-mode injection with negative absorber voltage, injected power ratio $\approx 2.1 \%$, the locking regions denoted by arrows (resolution bandwidth: $100 \mathrm{kHz}$ ). (a) Master laser's wavelength: $\lambda_{m}$. (b) Master laser's wavelength: $\lambda_{m}+0.015 \mathrm{~nm}(1.85 \mathrm{GHz})$. (c) Master laser's wavelength: $\lambda_{m}+0.024 \mathrm{~nm}(2.90 \mathrm{GHz})$. (d) Master laser's wavelength: $\lambda_{m}+0.028 \mathrm{~nm}(3.45 \mathrm{GHz})$.

\section{A. Wide RF locking-range}

We compared the RF locking-range in dual-mode injection for the two absorber voltages studied in $\S$ III. When injectionlocking occurred, the RF linewidth significantly narrowed forming a delta-like RF lineshape (beyond the resolution limit of our electrical spectrum analyzer, $1 \mathrm{kHz}$ ), similar to hybrid mode-locking, with reduced noise level. This resulted in significant reduction of timing jitter by more than one order of magnitude ( $\approx 235 \mathrm{fs}$ ) [18], [23]. The RF locking-range is defined here as the spectral region where strong narrowing of the RF linewidth occurred accompanied by a lowering of the noise level by $>30 \mathrm{~dB}$ (resolution bandwidth: $100 \mathrm{kHz}$ ). In the presence of any sidebands, possibly due to self-phase modulation, an additional criterion of side band suppression by more than $>30 \mathrm{~dB}$ was also applied. Similar to $\mathrm{CW}$ optical injection, the $20 \mathrm{~dB}$ suppression of free-running RF peak was applied for unlocked-locked transition as well. An example of such wide RF locking-range for negative absorber voltage is shown as intensity graphs in Figs. 7(a)-(d). The frequency spacing and wavelengths of the dual-modes were designed initially to match the RF peak of $\mathrm{CW}$ injection-locked case. RF locking in this regime (Fig. 7(a)) was limited to a range of 92 $\mathrm{MHz}$. However, by slightly increasing the master laser wavelength (center wavelength of the dual-modes) and keeping the RF spacing fixed, the slave laser transitioned from unlocked to injection-locked. Then, by increasing the frequency of the RF source, new frequencies could be included in the second locking region (Fig. 7(b)). Following this method, more locked regions were found, thereby widening the effective RF lockingrange significantly.

RF locking-ranges at various injected powers available in our experiment showed significant difference for negative and positive absorber voltages. The RF locking-range versus injected power ratio for two absorber voltages (positive: filled circles, negative: filled triangles) is shown in Fig. 8. As can be seen, the difference in locking-ranges for these cases are considerable due to the existence of several overlapping 
locked regions which broaden the upper bound of the total effective RF locking-range. The maximum RF locking-range for negative absorber voltage was $\approx 400 \mathrm{MHz}$ corresponding to injection ratio of $\approx 5 \%$. Extra locked regions were mostly absent or existed with much lower RF locking-ranges at positive absorber voltage except for highest available injection power (last two points in Fig. 7), with maximum injected power at port 2 of the circulator $\approx 330 \mu \mathrm{W}$. It is worth noting that for negative absorber voltage locking-ranges $>100 \mathrm{MHz}$ occurred at even small amounts of injected power.

Combining both frequency ranges covered by the injectionlocked laser for the two absorber voltages, one can extend the overall locking-range to $440 \mathrm{MHz}$, as shown in Fig. 9. A typical cleaving error of $20 \mu \mathrm{m}$ gives a device-to-device variation in designed repetition rate of $\sim 200 \mathrm{MHz}$ which can be covered easily by such a wide RF locking-range. This wide locking-range also included $21.33 \mathrm{GHz}$, locking to the subharmonic of $42.66 \mathrm{GHz}$ a defined frequency used in optical communication systems. This is $>300 \mathrm{MHz}$ higher than that of the free-running laser with negative absorber $(21.026 \mathrm{GHz})$ and $>470 \mathrm{MHz}$ from free-running positive absorber (20.858 $\mathrm{GHz}$ ).

The total RF locking-range of $440 \mathrm{MHz}$ for a $21 \mathrm{GHz}$ device means the tuning percentage of more than $2 \%$ is higher than the record published by monolithic hybrid modelocked laser at $1.55 \mu \mathrm{m}$ [13], [14] and comparable to the widest ranges reported in monolithic actively mode-locked lasers [15]. Moreover, these were achieved with special RF impedance matching circuits plus multi-section devices to extend the locking-range. Here we used simple two-section lasers without any mechanism for extra RF or phase tuning such as a passive section. Also, the optical nature of this synchronization mechanism makes this technique transparent against RF spectrum of injected signal, unlike hybrid/active mode-locking where impedance matching is crucial to increase the actual amount of injected RF power versus frequency. As we will show, injection-locking to a dual-mode spacing at higher harmonics of the repetition rate is also possible using this technique. Also, the shift of repetition rate observed in dual-mode injection versus free-running values gives an extra degree of freedom in frequency coverage, where a separate frequency range could be covered using hybrid mode-locking.

\section{B. Optical spectral properties}

In addition to the RF properties of the comb, optical spectral properties such as modal linewidth, comb spectral width and pulsing characteristics are important parameters that need to be considered in real applications. As mentioned earlier, an important advantage of dual-mode injection over electronic synchronization techniques such as hybrid mode-locking is the modal linewidth narrowing as a result of optical injectionlocking. In Fig. 10(a), the heterodyne beating tone of the several modes in different locations of the dual-mode injectionlocked comb (solid-lines) and free-running 20th mode away from second injected mode (dashed-line/blue) are shown. As can be seen, a strong narrowing occurred for all modes when the laser was injection-locked whereas the free running optical

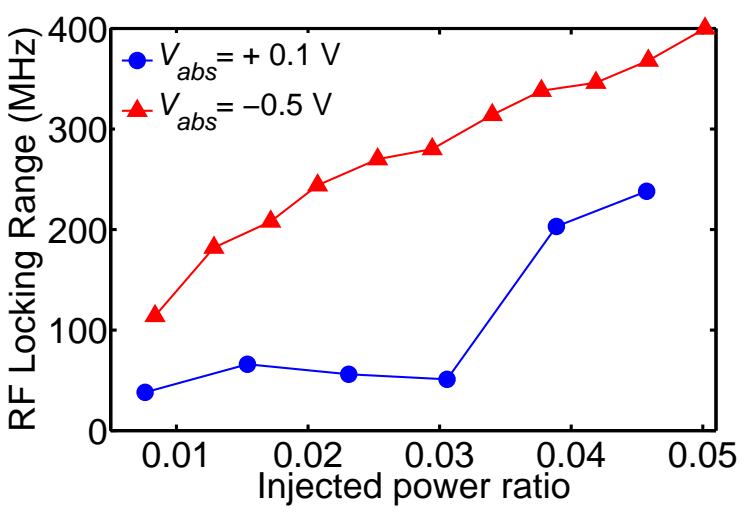

Fig. 8. RF locking-range for dual-mode injection versus injected power for positive absorber voltage (filled circles) and negative absorber voltage (filled triangles) and the same bias parameters as Fig. 3(a).

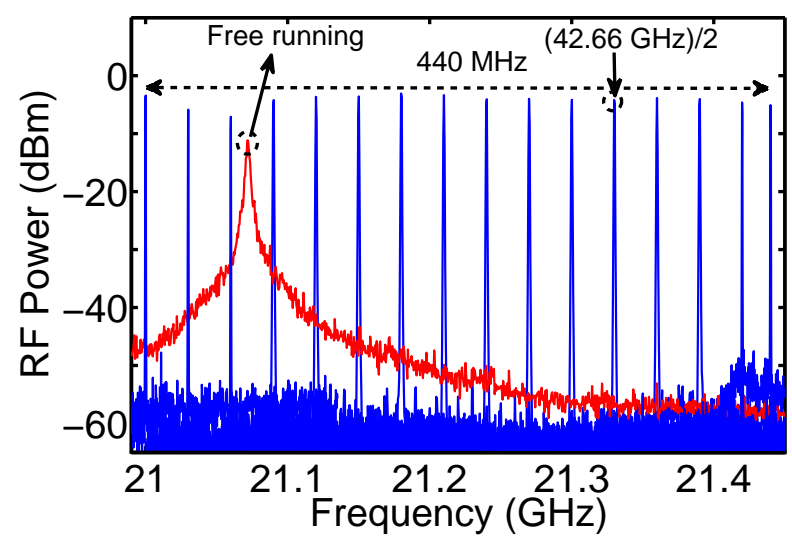

Fig. 9. Demonstration of wide $(\approx 440 \mathrm{MHz})$ repetition rate tuning by combining the locking-ranges for negative and positive absorber voltages. The RF spectrum of the free-running mode-locked laser is shown for comparison.

linewidth was $\approx 270 \mathrm{MHz}$. The normalized lineshape of this mode (solid-line/red) along with the lineshape of one of the master laser's modulated sidebands (dashed-line/black) is given in Fig. 10(b). It is evident that the lineshape of the measured mode follows that of the master laser which is a clear indication of complete coherence of this mode and the dual-modes. As shown later, this characteristic is very useful for applications requiring comb generation with optical phase synchronization of the comb lines to the master laser.

The width of pulses generated by the dual-mode injectionlocked comb, and $10 \mathrm{~dB}$ spectral width versus locked frequency for two injection powers are shown in Fig. 11. The pulse-width (FWHM of $\mathrm{Sech}^{2}$ fit to the autocorrelation trace after deconvolution) of the comb started from 3.4 and 3.7 ps for injection ratio of $2.9 \%$ and $4.6 \%$, respectively. As the frequency spacing of the comb increased within the lockingrange, the pulses broadened monotonically up to $\approx 6 \mathrm{ps}$. The increase in the pulse-width was followed by decrease of the 10 $\mathrm{dB}$ comb spectral width. The number of comb lines within 10 $\mathrm{dB}$ of the peak started at 35 and 31 for the injection ratios of $2.9 \%$ and $4.6 \%$, respectively;then, decreased as the frequency increased with final counts 13 and 11 lines within $10 \mathrm{~dB}$.

Finally, the optical spectra of the comb at selected locking 


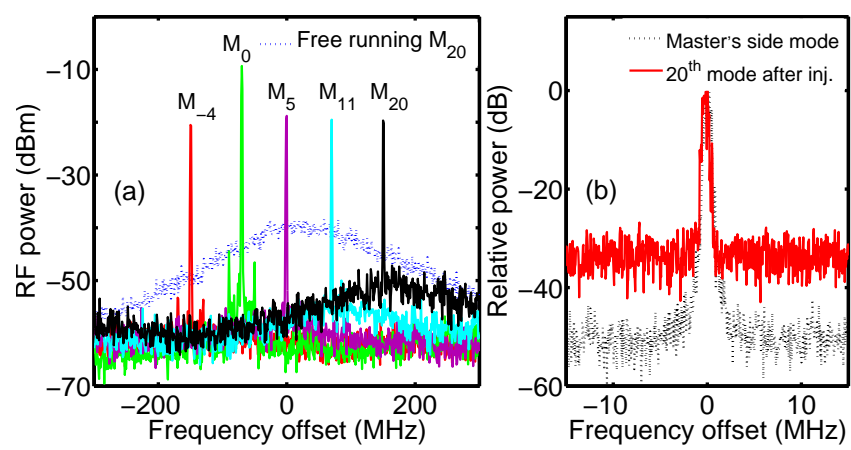

Fig. 10. (a). Heterodyne beat note of several modes at different locations of the OFC (solid-lines) along with free running (dashed-line/blue) 20th mode away from second injected mode. The modes are denoted based on their relative modal distance from injected modes; mode with negative indice located at the blue side of injection. (b). Zoom (30 MHz span) of the normalized RF beat note for the mode (solid-line/red) and one of the master's modulated side bands (dashed-line/black), injected power ratio $\approx 3 \%$.

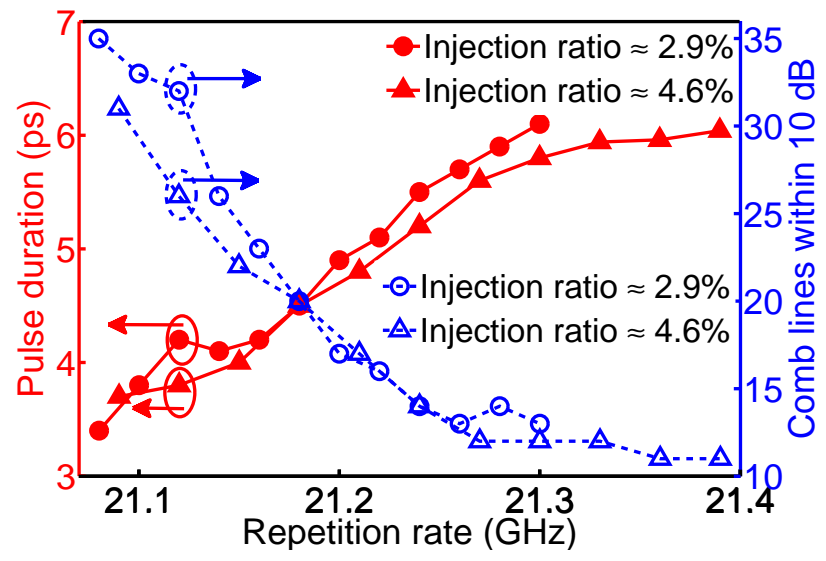

Fig. 11. Comb pulse-width (filled marks, left axis) and number of comb lines within $10 \mathrm{~dB}$ spectral width (empty marks, right axis) versus repetition rate of injection-locked laser $\left(f_{r e p}\right)$ for injection ratios of $\approx 2.9 \%$ and $\approx 4.6 \%$ ).

frequencies separated by $60 \mathrm{MHz}$ from Fig. 11 is shown in Fig. 12. The general shape of the comb consisted of two prominent modes at the location of injection, followed by an almost flat range and a group of few modes having the highest powers among the comb lines in the red side of the comb. This is similar to the red shift phenomenon observed in GaAs quantum-dot mode-locked lasers under optical injection [23]. As the frequency increased, the spectral distance of this part and the injected modes decreased, forming a comb with a narrower $10 \mathrm{~dB}$ width. This was also followed by presence of lines with unequal power at the location of injection despite the fact that the dual-modes had same power before injection. The comb's spectral width could be further expanded using an optical amplifier and highly nonlinear fibre to be tailored to a particular application [11]. The autocorrelation traces with their Sech $^{2}$ fit and transform limited autocorrelation traces for the same injection parameters as Fig. 12 are shown in Fig. 13. The transform limited traces were calculated from the measured optical spectra assuming zero chirp or constant spectral phase. The calculated time-bandwidth product was 1.53 for the widest optical spectrum decreasing to 0.72 for
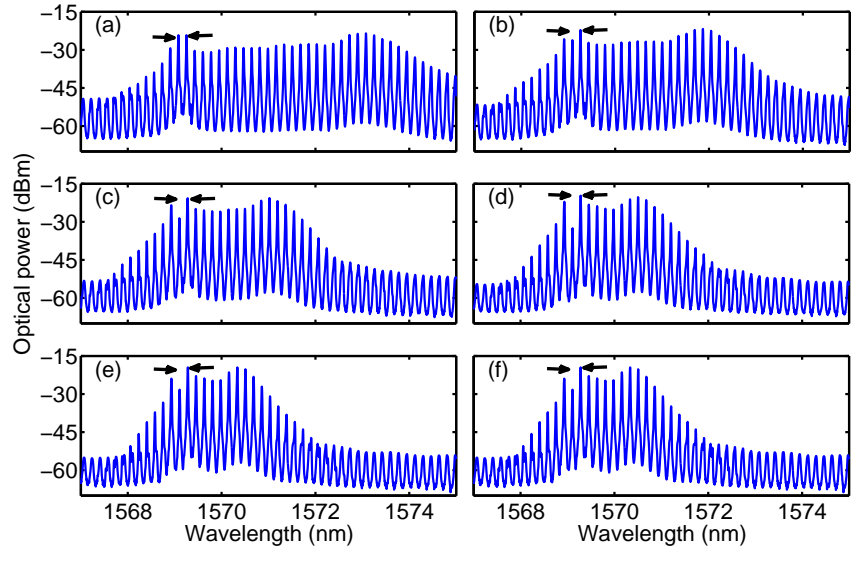

Fig. 12. Optical spectrum of OFC for injection ratio of $\approx 4.6 \%$ at selected frequencies with the step of $60 \mathrm{MHz}$ corresponding to Fig. 11. (a) $f_{r e p}=$ $21.09 \mathrm{GHz}$. (b) $f_{r e p}=21.15 \mathrm{GHz}$. (c) $f_{r e p}=21.21 \mathrm{GHz}$. (d) $f_{r e p}=21.27$ GHz. (e) $f_{r e p}=21.33 \mathrm{GHz}$. (f) $f_{r e p}=21.39 \mathrm{GHz}$. The arrows denote the spectral location of injected dual-modes.
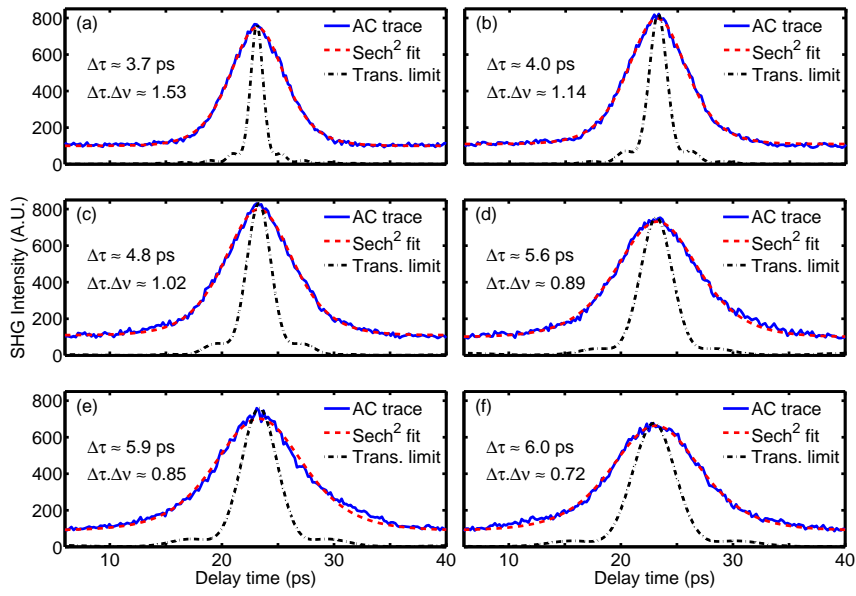

Fig. 13. Autocorrelation traces (blue/solid-lines) along with $\mathrm{Sech}^{2}$ fit (red/dashed-line) and transform limited autocorrelation traces (black/dasheddot line) corresponding to the optical spectra in Fig. 12

the narrowest spectrum indicating residual chirp for injectionlocked laser. The free-running time-bandwith products were 7.17 for positive and 2.83 for negative absorber bias indicating reduction of time-bandwidth product with optical injection.

\section{PhASE-SYNCHRONIZATION OF A TWO-CHANNEL PHASE SENSITIVE AMPLIFIER USING DUAL-MODE INJECTION}

Phase sensitive amplifiers (PSAs) have recently attracted a lot of interest due to their unique phase squeezing capabilities, which makes them an ideal technology platform for regenerating phase encoded modulation formats, e.g. differential phase shift keying (DPSK) [24]. Although their phase squeezing capabilities have been well known for more than a decade [25], a practical implementation that would allow them to stand as independent, black box elements in transmission links was missing until recently [26]. This was achieved using the synchronization scheme proposed in [27], which combined carrier extraction and frequency generation functionalities along with optical injection-locking of a local oscillator laser 


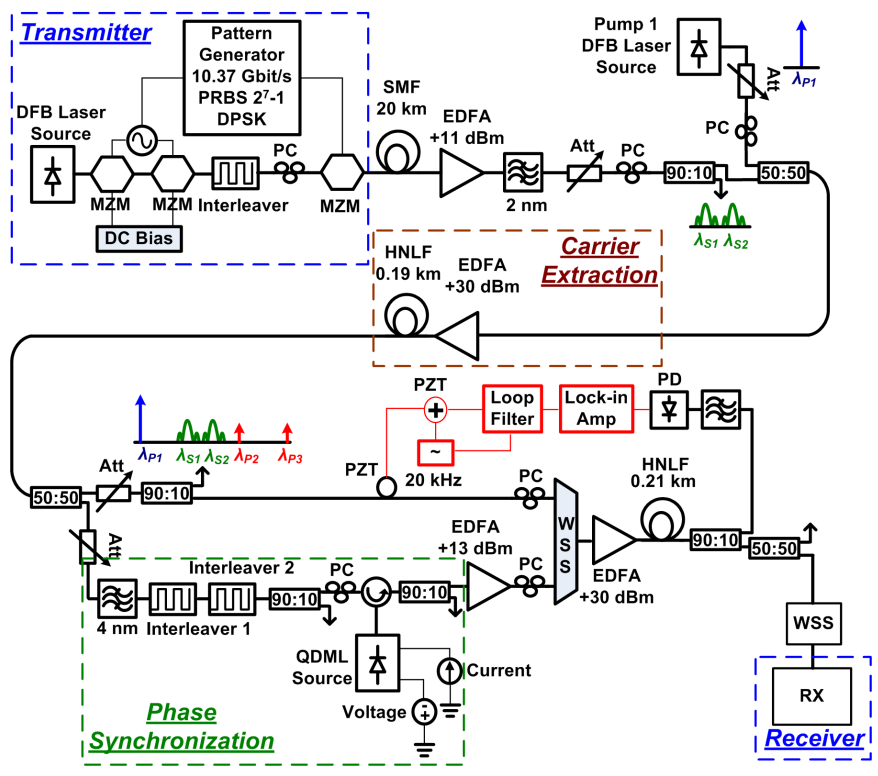

Fig. 14. Experimental arrangement for two-channel DPSK PSA based on QDML phase synchronization. List of abbreviations used: MZM: MachZehnder Modulator, PC: Polarization Controller, SMF: Single-Mode Fibre, Att. Variable Optical Attenuator, HNLF: Highly Nonlinear Fibre, PZT: PiezoElectric Transducer, PD: Photodetector, WSS: Wavelength Selective Switch, RX: Receiver.

to create the synchronized pumps of the PSA. Recently, this scheme was extended to demonstrate the first two-channel DPSK PSA using optical injection-locking of two independent single-mode lasers [28].

However, increasing the number of channels in such a multi-pump black box PSA, requires at its synchronization stage an equal number of additional local pumps, each one of them phase-locked to the corresponding extracted carrier. This makes the overall setup complicated and bulky, effectively undermining the multi-wavelength processing capability of such regenerators. Therefore, a solution that would enable multiple pump generation by synchronizing a single laser comb-source would be advantageous. In this section, we demonstrate a powerful application of the dual-mode injectionlocking technique described in $\S \mathrm{IV}$, which allowed us to create multiple synchronized pumps for phase sensitive amplification using a single QDML which was injection-locked by two extracted signal carriers.

\section{A. Experimental arrangement and results}

The experimental set up is illustrated in Fig. 14. At the transmitter a comb generator consisting of two cascaded Mach-Zehnder modulators was used to create synchronized optical carriers spaced at $41.496 \mathrm{GHz}$. Two of them were selected at $\lambda_{s 1}=1558.431 \mathrm{~nm}$ and $\lambda_{s 2}=1558.759 \mathrm{~nm}$ and modulated in DPSK format at $10.374 \mathrm{Gbit} / \mathrm{s}$ with a pseudorandom binary sequence (PRBS) of $2^{7}-1$. Subsequently, the channels were decorrelated by $20 \mathrm{~km}$ of conventional single-mode fiber (SMF) and amplified by an EDFA at 11 $\mathrm{dBm}$. At the carrier extraction stage of the PSA, two input signals were mixed with a local DFB laser emitting at $\lambda_{P 1}=1554.898 \mathrm{~nm}$, then amplified to $30 \mathrm{dBm}$ in a highly

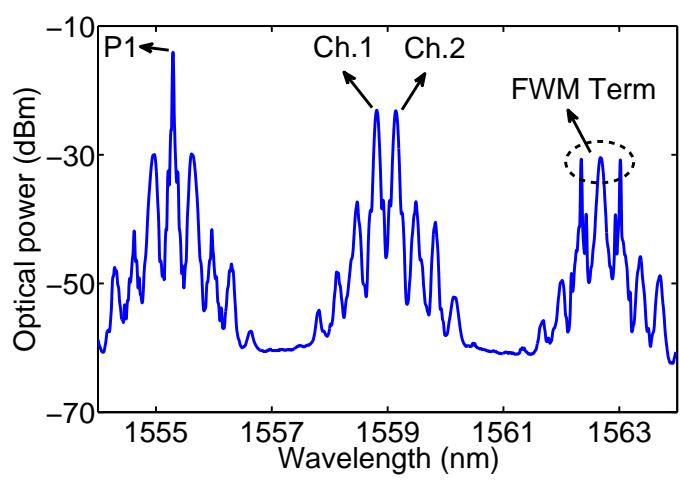

Fig. 15. Optical spectrum of first HNLF output. The arrows denote the two DPSK channels (Ch.1, Ch.2), the local pump (P1), and the pumps generated through FWM process.

nonlinear fiber (HNLF). The HNLF had a length of $0.198 \mathrm{~km}$, dispersion parameter $-0.2 \mathrm{ps} /(\mathrm{nm} . \mathrm{km})$ (at $1550 \mathrm{~nm})$, nonlinear coefficient $7.4 \mathrm{~W}^{-1} \cdot \mathrm{km}^{-1}$ and attenuation of $14 \mathrm{~dB} / \mathrm{km}$ [29]. At the HNLF output the two generated carriers were selflocked to the corresponding signals and the local pump and located at symmetric wavelengths with respect to them, as shown in Fig. 15. The output of the HNLF was divided in two arms. In one arm, we used a band pass filter $(3 \mathrm{~dB}$ bandwidth $4 \mathrm{~nm}$ ) and two interleavers $(50 \mathrm{GHz}$ and 21.31 $\mathrm{GHz}$, respectively) to select the carriers and clean them from the residual frequencies of the FWM process. Then, the filtered spectrum was injected through a circulator to the QDML. The circulator was connected to the QDML through a lensed fibre introducing a typical loss of $3 \mathrm{~dB}$. The output of the laser from port 3 of the circulator (average power $\approx-1.2 \mathrm{dBm}$ ) was then amplified to $13 \mathrm{dBm}$ in an EDFA and combined with the pump and signal spectrum of the other arm to a Wavelength Selective Switch (WSS). The WSS also removed unwanted frequencies from the carrier extraction process and selected the two signal channels and the three pumps, as shown in Fig. 16(a) along with the optical spectrum of injection-locked QDML. The output of WSS was amplified by a $30 \mathrm{dBm}$ high power EDFA before launched to a second HNLF (with the same parameters as first HNLF and with a length of 0.19 $\mathrm{km})$ to form the PSA. We used a conventional feedback based

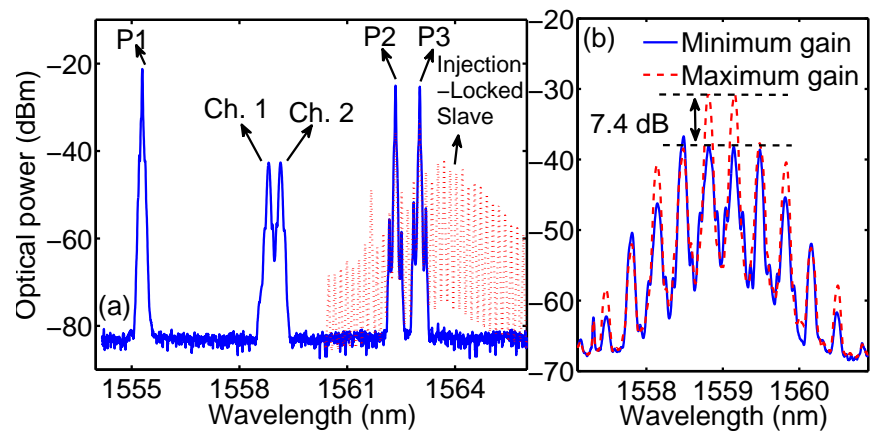

Fig. 16. (a) Optical spectrum of PSA input, after removing un-wanted components using WSS, the spectrum of OFC from QDML is depicted in dashed curve. (b). The zoom of the optical spectrum at the PSA output around channels for maximum (dashed line-red) and minimum (solid-line/blue) gain. 
phase-locked loop circuit to control a piezoelectric-based fibre stretcher (PZT) and compensate any slow thermal or acoustic induced phase drift between the interacting waves in both paths. The stabilized output of the PSA against thermal fiber expansion was observed at maximum and minimum gain for both channels, as shown in Fig. 16(b). A 7.4 dB on-off gain was measured for both channels which may be increased by using higher pump powers.

The QDML used in this experiment was a two-section device with total cavity length of $2030 \mu \mathrm{m}$, with $40 \mu \mathrm{m}$ saturable-absorber section (2\%) operating as self-mode-locked (SML) lasers [30] when the absorber was left floating, giving mode-locked frequency around $20.8 \mathrm{GHz}$ and the same mounting configuration as described in $\S$ II.

The spacing of extracted carriers was twice of that the channels due to FWM process; this means injection at the 4th harmonic of the repetition rate of the injection-locked laser $(82.992 \mathrm{GHz})$. Nevertheless, the OFC with the same line spacing as dual-mode injection at first harmonic was generated as the locking mechanism is not related to any possible FWM process in the QDML's cavity [31]. The RF spectrum of the laser when free running (without injection) and under injection-locking is depicted in Fig. 17, where significant RF linewidth narrowing was observed. The Lorentzian fit to the RF linewidth of the free running laser was $270 \mathrm{kHz}$ (not shown) whereas the RF linewidth of the injection-locked laser reduces to the values beyond the resolution bandwidth (RBW) of the instrument. The inset in Fig. 17 shows the $80 \mathrm{~Hz}$ zoom of the peak with resolution bandwidth of $1 \mathrm{~Hz}$; the 20 $\mathrm{dB}$ linewidth of the tone is estimated to be $3 \mathrm{~Hz}$, indicating strong reduction of timing jitter of the laser. This confirms the presence of phase-locking between the injected modes and extracted carriers.

Another difference between the dual-mode injection here to that described in $\S$ IV comes from the generation mechanism of coherent dual-modes. In $\S$ IV, the modes were generated from a $\mathrm{CW}$ source with high optical signal to noise ratio (OSNR) which indicates dual-modes with high OSNR. In the case of dual-modes generated through FWM in a HNLF, however, any noise present in signals and EDFA will be transferred to the generated carriers. Therefore, special care has to be taken to make sure that the dual-modes are injected with OSNR higher than a threshold to have complete injectionlocking. Correct evaluation of this threshold requires a separate study to investigate the robustness of dual-mode injection scheme against OSNR similar to what has been done in [27] for injection locking to $\mathrm{CW}$ slave lasers. In addition to the noise, residual phase modulation is present in the generated carriers. In order to suppress the residual phase modulation, the injection ratio should be kept as low as possible [32] (about $-30 \mathrm{~dB}$ in this experiment).

Both QDMLs with SAML or SML mechanisms could be used for injection-locking to the extracted carriers. OFCs derived from SMLs have fewer comb lines with the majority of the power in the injected modes. Combs from SAMLs are much wider, with power distributed among many comb lines (see Fig. 12). SML combs are beneficial for this scheme, as more power per line was required to observe phase sensitive

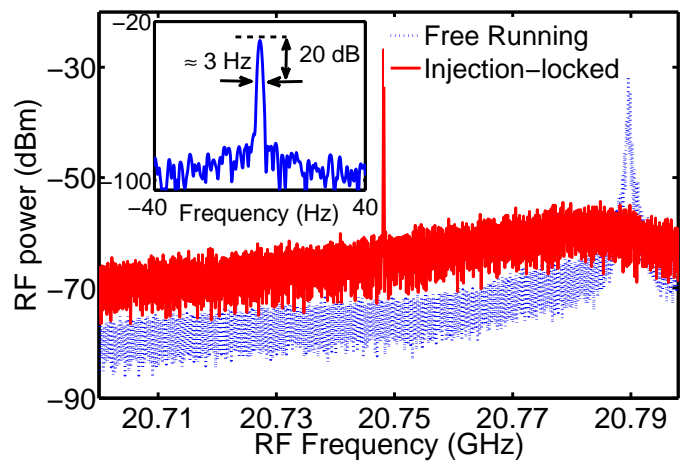

Fig. 17. RF spectrum of free running (dashed-line/blue) and injection-locked QDML (solid-line/red), the inset shows a zoom of the RF tone with $80 \mathrm{~Hz}$ span and RBW of $1 \mathrm{~Hz}$. the $20 \mathrm{~dB}$ linewidth is $\approx 3 \mathrm{~Hz}$.

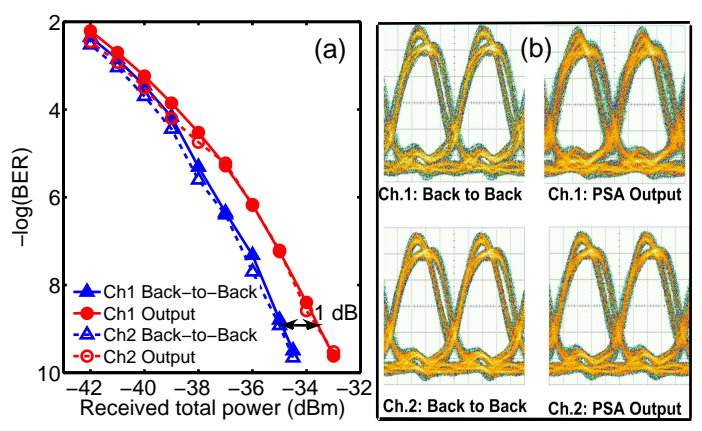

Fig. 18. (a). BER measurements of both channels versus total received power. (b). Error free eye diagrams for both channels at the input and output of the PSA.

performance at the output of the PSA.

We also studied the performance of the PSA in terms of BER measurements for both channels. Fig. 18(a) depicts the BER for both channels in back-to-back and PSA output (maximum gain) against received power. By comparing the BER curves for both channels at the output, about $1 \mathrm{~dB}$ sensitivity penalty for BER of $10^{-9}$ was observed, with negligible penalty at the forward error correction (FEC) threshold around $10^{-3}$. The error-free eye diagrams (shown in Fig. 18(b) for both channels at the output are open proving the excellent performance of our scheme.

\section{CONCLUSION}

In this paper, we reported the generation of versatile narrow linewidth optical frequency combs with wide spectra, based on dual-mode injection-locking of InP quantum-dash modelocked lasers. A wide RF locking-range of $\approx 440 \mathrm{MHz}$ was obtained for a two-section device without any passive section for extra tuning. This is $>2 \%$ of repetition rate, higher than the records published for any monolithic hybrid mode-locked laser. It was shown that proper selection of bias parameters led to such a wide RF locking-range. This behavior is believed to be due to injection-locking to the regime of less stable mode-locking operation opening up wide locking-range and repetition rate tuning. The widest comb had $10 \mathrm{~dB}$ spectral width of more than $0.7 \mathrm{THz}$ (35 lines $\approx 21 \mathrm{GHz}$ spacing) 
with 3.7 ps pulses. The individual comb lines, all showed similar linewidths as the master laser. A two-channel PSA with two $10.374 \mathrm{~Gb} / \mathrm{s}$ DPSK input channels was demonstrated based on simultaneous phase synchronization of local pumps to incoming signals through this novel injection-locking technique using a $21 \mathrm{GHz}$ InP QDML. A $7.4 \mathrm{~dB}$ phase sensitive gain and only $1 \mathrm{~dB}$ sensitivity penalty observed in the BER measurements of two channels at the output of the amplification stage. This scheme has the potential for extension to multiple channels towards more compact and efficient black box PSAs.

\section{ACKNOWLEDGMENT}

The authors would like to thank Dr. R. J. Manning for critical reading of this manuscript.

\section{REFERENCES}

[1] A. Sano, Y. Takatori, and Y. Miyamoto, "No-guard-interval coherent optical OFDM for 100-Gb/s/ch long-haul transmission systems," in Proc. Optical Fiber Communication Conference, OFC 2009, 2009,, p. Paper OTuO3.

[2] A. Ellis and F. Gunning, "Spectral density enhancement using coherent WDM," IEEE Photon. Technol. Lett., vol. 17, no. 2, pp. 504-506, Feb. 2005.

[3] T. Yilmaz, C. DePriest, T. Turpin, J. Abeles, and P. Delfyett Jr, "Toward a photonic arbitrary waveform generator using a modelocked external cavity semiconductor laser," IEEE Photon. Technol. Lett., vol. 14, no. 11, pp. 1608-1610, Nov. 2002.

[4] D. Miyamoto, K. Mandai, T. Kurokawa, S. Takeda, T. Shioda, and H. Tsuda, "Waveform-controllable optical pulse generation using an optical pulse synthesizer," IEEE Photon. Technol. Lett., vol. 18, no. 5, pp. $721-723$, Mar. 2006

[5] C. Jáuregui Misas, P. Petropoulos, and D. Richardson, "All-optical signal processing of periodic signals using a brillouin gain comb," J. Lightw. Technol., vol. 26, no. 17, pp. 3110-3117, Sep. 2008.

[6] S. Fukushima, C. Silva, Y. Muramoto, and A. Seeds, "Optoelectronic millimeter-wave synthesis using an optical frequency comb generator, optically injection locked lasers, and a unitraveling-carrier photodiode,' J. Lightw. Technol., vol. 21, no. 12, pp. 3043-3051, Dec. 2003.

[7] T. Healy, F. C. G. Gunning, A. D. Ellis, and J. D. Bull, "Multiwavelength source using low drive-voltage amplitude modulators for optical communications," Opt. Express, vol. 15, no. 6, pp. 2981-2986, Mar. 2007.

[8] W. Mao, P. Andrekson, and J. Toulouse, "Investigation of a spectrally flat multi-wavelength DWDM source based on optical phase- and intensitymodulation," in Proc. Optical Fiber Communication Conference, OFC 2004, Feb. 2004, Paper MF 78.

[9] H. Y. Ryu, H. S. Moon, and H. S. Suh, "Optical frequency comb generator based on actively mode-locked fiber ring laser using an acousto-optic modulator with injection-seeding," Opt. Express, vol. 15, no. 18, pp. 11396-11 401, Sep. 2007.

[10] I. Ozdur, M. Akbulut, N. Hoghooghi, D. Mandridis, S. Ozharar, F. Quinlan, and P. Delfyett, "A semiconductor-based 10-GHz optical comb source with sub 3-fs shot-noise-limited timing jitter and 500-Hz comb linewidth," IEEE Photon. Technol. Lett., vol. 22, no. 6, pp. $431-433$, Mar. 2010.

[11] P. Anandarajah, R. Maher, Y. Xu, S. Latkowski, J. O'Carroll, S. Murdoch, R. Phelan, J. O'Gorman, and L. Barry, "Generation of coherent multicarrier signals by gain switching of discrete mode lasers," IEEE Photon. J., vol. 3, no. 1, pp. $112-122$, Feb. 2011.

[12] T. Jung et al., "CW injection locking of a mode-locked semiconductor laser as a local oscillator comb for channelizing broad-band RF signals," IEEE Trans. Microw. Theory Tech., vol. 47, no. 7, pp. 1225 -1233, Jul. 1999.

[13] S. Arahira, N. Mineo, K. Tachibana, and Y. Ogawa, "40 GHz hybrid modelocked laser diode module operated at ultra-low RF power with impedance-matching circuit," Electron. Lett., vol. 39, no. 3, pp. 287 289, Feb. 2003.

[14] R. Kaiser and B. Huttl, "Monolithic 40-GHz mode-locked MQW DBR lasers for high-speed optical communication systems," IEEE J. Sel. Topics Quantum Electron., vol. 13, no. 1, pp. 125-135, Jan./Feb. 2007.
[15] S. Arahira and Y. Ogawa, "40 ghz actively mode-locked distributed bragg reflector laser diode module with an impedance-matching circuit for efficient RF signal injection," Jpn. J. Appl. Phys, vol. 43, no. 4B, pp. 1960-1964, 2004.

[16] W. Lee and P. Delfyett, "Dual-mode injection locking of two independent modelocked semiconductor lasers," Electron. Lett., vol. 40, no. 19, pp. 1182-1183, Sep. 2004.

[17] F. Lelarge et al., "Recent advances on InAs/InP quantum dash based semiconductor lasers and optical amplifiers operating at $1.55 \mu \mathrm{m}$," IEEE J. Sel. Topics Quantum Electron., vol. 13, no. 1, pp. 111-124, 2007.

[18] E. Sooudi, G. Huyet, J. McInerney, F. Lelarge, K. Merghem, R. Rosales, A. Martinez, A. Ramdane, and S. Hegarty, "Injection-locking properties of InAs/InP-based mode-locked quantum-dash lasers at $21 \mathrm{GHz}$," IEEE Photon. Technol. Lett., vol. 23, no. 20, pp. 1544 -1546, Oct. 2011.

[19] R. Rosales, K. Merghem, A. Martinez, A. Akrout, J.-P. Tourrenc, A. Accard, F. Lelarge, and A. Ramdane, "InAs/InP quantum-dot passively mode-locked lasers for 1.55- $\mu \mathrm{m}$ applications," IEEE J. Sel. Topics Quantum Electron., vol. 17, no. 5, pp. 1292 -1301, 2011.

[20] H. Haus, "Theory of mode locking with a slow saturable absorber," IEEE J. Quantum Electron., vol. 11, no. 9, pp. 736 - 746, Sep. 1975.

[21] S. Arahira and Y. Ogawa, "Repetition-frequency tuning of monolithic passively mode-locked semiconductor lasers with integrated extended cavities," IEEE J. Quantum Electron., vol. 33, no. 2, pp. 255 -264, Feb. 1997.

[22] N. Rebrova, G. Huyet, D. Rachinskii, and A. G. Vladimirov, "Optically injected mode-locked laser," Phys. Rev. E, vol. 83, p. 066202, Jun. 2011.

[23] T. Habruseva, S. O'Donoghue, N. Rebrova, D. Reid, L. Barry, D. Rachinskii, G. Huyet, and S. Hegarty, "Quantum-dot mode-locked lasers with dual-mode optical injection," IEEE Photon. Technol. Lett., vol. 22, no. 6, pp. $359-361$, Mar. 2010.

[24] K. Croussore and G. Li, "Phase and amplitude regeneration of differential phase-shift keyed signals using phase-sensitive amplification," IEEE J. Sel. Topics Quantum Electron., vol. 14, no. 3, pp. 648 -658, 2008.

[25] M. Marhic, C. Hsia, and J.-M. Jeong, "Optical amplification in a nonlinear fibre interferometer," Electron. Lett., vol. 27, no. 3, pp. 210 -211 , Jan. 1991.

[26] R. Slavik et al., "All-optical phase and amplitude regenerator for nextgeneration telecommunications systems," Nat. Photon., vol. 4, pp. 690 $-695,2010$.

[27] R. Weerasuriya, S. Sygletos, S. Ibrahim, R. Phelan, J. O'Carroll, B. Kelly, J. O'Gorman, and A. Ellis, "Generation of frequency symmetric signals from a BPSK input for phase sensitive amplification," in $O P$ tical Fiber Communication (OFC), 2010 Conference on (OFC/NFOEC), Mar. 2010,, p. Paper OWT6.

[28] S. Sygletos, P. Frascella, S. K. Ibrahim, L. Grüner-Nielsen, R. Phelan, J. O'Gorman, and A. D. Ellis, "A practical phase sensitive amplification scheme for two channel phase regeneration," Opt. Express, vol. 19, no. 26, pp. B938-B945, Dec. 2011.

[29] L. Gruner-Nielsen et al., "A silica based highly nonlinear fibre with improved threshold for stimulated brillouin scattering," in Optical Communication (ECOC), 2010 36th European Conference and Exhibition on, Sep. 2010.

[30] E. Sooudi, G. Huyet, J. McInerney, F. Lelarge, K. Merghem, A. Martinez, A. Ramdane, and S. Hegarty, "Observation of harmonic-modelocking in a mode-locked inas/inp-based quantum-dash laser with $\mathrm{cw}$ optical injection," IEEE Photon. Technol. Lett., vol. 23, no. 9, pp. 549 -551 , May 2011.

[31] T. Habruseva, G. Huyet, and S. Hegarty, "Dynamics of quantum-dot mode-locked lasers with optical injection," IEEE J. Sel. Topics Quantum Electron., vol. 17, no. 5, pp. $1272-1279,2011$.

[32] S. Sygletos, S. K. Ibrahim, R. Weerasuriya, R. Phelan, L. G. Nielsen, A. Bogris, D. Syvridis, J. O'Gorman, and A. D. Ellis, "Phase synchronization scheme for a practical phase sensitive amplifier of ASK-NRZ signals," Opt. Express, vol. 19, no. 13, pp. 12 384-12 391, Jun. 2011. 


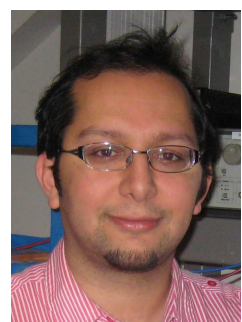

Ehsan Sooudi (S'10) received his B.Sc. degree in electrical engineering from Sharif University of Technology, Tehran, Iran and his M.Sc. degree in electrical engineering from Tarbiat Modares University, Tehran, Iran, in 2003 and 2006, respectively. $\mathrm{He}$ is currently working towards his Ph.D. degree in Physics at University College Cork and Tyndall National Institute, Cork, Ireland. His research interests include quantum dash/dot mode-locked lasers, optical injection-locking, frequency comb generation, and applications of mode-locked lasers in optical

communication systems.

Stylianos Sygletos received the diploma degree in electrical engineering and the Ph.D. in 2005 working on the physical layer design of high capacity metropolitan area networks from the National Technical University of Athens (NTUA), Athens, Greece.

In 2006, he joined Athens Information Technology (AIT), Greece, where he performed research on impairment constraint based routing and developed simulation tools for quantum dot semiconductor optical amplifiers (QDSOAs). One year later, he joined the Institute of High Frequency and Quantum Electronics (IHQ) at the University of Karlsruhe. There, he continued his work on QD-SOAs and developed appropriate optical filtering schemes to support the high speed nonlinear performance of those devices. Currently he is with Tyndall Institute of Technology where he works in the development of optical phase locked loops for applications in coherent detection, phase sensitive amplification/regeneration and sampling. He is coauthor of more than 80 publications in international journals and conferences.

Andrew D. Ellis received a BSc honours degree in physics with a minor in mathematics from the University of Sussex in 1987 and a Ph.D degree in electronic engineering from the University of Aston in Birmingham in 1997. Between 1987 and 2000 he held several positions at BT Laboratories where he investigated long haul and submarine transmission systems. He joined the Corning Research centre in 2000 and became a Senior Research Fellow at University College Cork, Cork, Ireland in 2003 where he manages a research group investigating the advanced technologies for optical networks, including digital signal processing and optical super channels.

Prof Ellis has authored or co-authored over 150 international papers and has published over 20 patent families pertinent to optical communications. He has been a member of the technical program committee for the European Conference on Optical Communications (ECOC) since 2004, and has twice been a member of the technical program committee for the Optical Fibre Communications conference (OFC). He was appointed as an IEEE Photonics Society Distinguished Lecturer for the 2012/13 term.

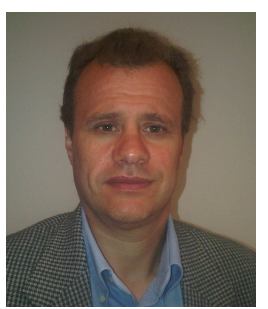

Guillaume Huyet (SM'00) received his M.Sc. degree from ENS Lyon, Lyon, France, and his Ph.D. degree from the University of Nice, Nice, France.

After postdoctoral positions in Mallorca, Spain, Cork, Ireland, and Glasgow, U.K., he became a Lecturer at University College, Cork, Ireland. In 2002, he received the Science Foundation Ireland Principal Investigator Award and became a Senior Research Fellow at University College, Cork, Ireland. He is currently the Science Foundation Ireland Principal Investigator at the Tyndall National Institute and Head of the Center of Advanced Photonics and Process Analysis (CAPPA), Cork Institute of Technology, Cork. He has published more than 100 papers in international journals. His research interests include semiconductor devices for applications in communications and life science.

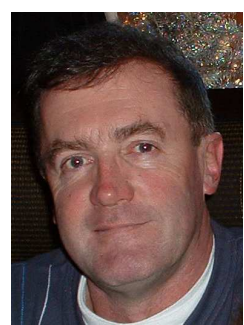

John G. McInerney is Professor and Head of Physics at University College Cork since 1993, and has led the laser physics group at the Tyndall National Institute in Cork, Ireland since its inception in 2004. He received a BSc from University College Cork and a PhD from Trinity College Dublin, both in Physics. He was a research engineer at Standard Telecommunication Labs, Harlow, England from 1981-2 and held the STL Research Fellowship in Optoelectronics at the Cavendish Laboratory, Cambridge University, from 1984-86, working on nanoscale gratings for photonics. From 1986-93 he was an assistant then associate professor of electrical and computer engineering at the University of New Mexico, Albuquerque, NM, working on high power and vertical cavity semiconductor lasers. From 2001-3 he was director of R\&D and new product realization at Novalux Inc, Sunnyvale, CA, leading development of high power frequency-doubled surface-emitting laser diodes. He is also an adjunct professor of optical sciences at the University of Arizona since 1993. His research interests include semiconductor laser dynamics, mode locking and pulse generation, mode control and stabilization, injection locking and external feedback effects, power scaling and high-brightness sources. He is a Fellow of the Institute of Physics and a member of OSA, SPIE and the IEEE Photonics Society.

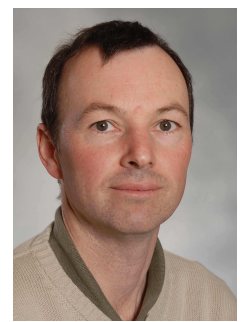

François Lelarge received the Diploma in material science in 1993 and the Ph. D degree in 1996, both from the University of Pierre et Marie Curie, Paris, France. From 1993 to 1996, he was with the Laboratory of Microstructures and Microelectronic, CNRS Bagneux, France. His thesis work was devoted to the fabrication and the optical characterization of GaAs/AlAs lateral supperlattice grown on vicinal surfaces by MBE. From 1997 to 2000, he was a postdoctoral Researcher at the Institute of Micro and Optoelectronics, Lausanne, Switzerland. He worked on InGaAs/GaAs quantum wires fabrication by MOCVD regrowth on patterned substrates. Presently, he is working within III-V Lab on InGaAsP/InP GSMBE growth for optoelectronic devices, in particular using QDot based active layers. He has published more than 110 papers and communications in national and international conferences, including 6 invited talks and 5 post-deadline papers on QDot based devices. He is author or co-author of more than 200 scientific papers.

He was in charge for III-VLab of the technical management of the Zodiac project (IST 2005-2008) on QDots based lasers and he is actually coordinator of two ANR projects on QD-based mode-locked laser (ANR-TELDOT) and directly modulated lasers (ANR-DIQDOT).

Kamel Merghem received Master's Degree in lasers and applications from Université des Sciences et Technologies, Lille, France in 2000. Since 2002, He is a research engineer at CNRS-Laboratory for Photonics and Nanostructures, Marcoussis, France. He is working on fabrication and characterization of semiconductor lasers for telecom applications. His current research interests include semiconductor mode-locked lasers and quantum dot lasers. He received a PhD degree in Physics from the University of Lille, in 2011. He has published over 30 papers in peer-reviewed journals.

Ricardo Rosales received the M.Sc. degree in Electrical and Optical Engineering from Telecom SudParis, France in 2009. He is currently working toward the Ph.D. degree at the CNRS-Laboratory for Photonics and Nanostructures, Marcoussis, France. His current research interests include semiconductor mode locked lasers and quantum-dot laser diodes. 
Anthony Martinez obtained his PhD in 2002 in Optoelectronics from the Laboratory for Optics P.-M. Duffieux, Institut Femto-ST. As a post-doc fellow at LPN (2003-2005), he was in charge of the design fabrication and characterizations of high speed lasers based on novel material systems: GaInNAs/GaAs QWs for low-cost uncooled lasers at $1.3 \mu \mathrm{m}$ within a precompetitive project, InAs/GaAs quantum dot material for application at $1.3 \mu \mathrm{m}$, InAs/InP (311)B QDots and InAs/InP quantum-dash mode-locked lasers (MLLs) at $1.55 \mu \mathrm{m}$ in collaboration with III-V Lab. He was a postdoc fellow during about a year (2005) in Pr. Lester's group (cofounder of Zia Laser Inc.) at the Center For High Technology Materials (NM) where he was involved in microwave properties of InAs/GaAs QD lasers, gain and absorption measurements of QD/QDash waveguides and InAs/GaAs QD superluminescent diodes. He was hired as a permanent researcher at LPN in 2006, he was involved in QD/Qdash based lasers and amplifiers for telecom applications within the framework of an European Integrated project ZODIAC, two European Networks of Excellence SANDie and EPIXnet. His current research activities include photonics components, i.e. semiconductor based lasers and amplifiers based on novel material systems, for telecommunication and defence applications within the framework of a French national project ANR Teldot and a FP7 Initial Training Network Prophet. He has published over 30 papers in peer-reviewed journals and is the author of a chapter in a book on QD MLLs and their applications.

Abderrahim Ramdane received a $\mathrm{PhD}$ degree in Semiconductor Physics from the University of Nottingham, in 1981, followed by a two-year fellowship to investigate deep levels in III-V compounds. In 1983 he was appointed Head of the Solar Material Laboratory in Algiers, investigating single crystal and amorphous silicon solar cells. In 1990 he joined FRANCE TELECOM / Centre National d'Etudes des Télécommunications (CNET Bagneux), in charge of the "Photonic Integrated Circuits on InP" activity. Since 1999 he has been a 'Directeur de Recherche' at CNRS-Laboratory for Photonics and Nanostructures working on nanostructured optical devices. He has authored or co-authored over 200 publications in peer reviewed journals and international conferences and holds 5 patents in the field of optoelectronic devices.

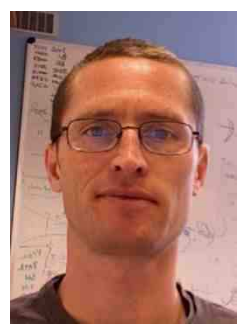

Stephen P. Hegarty (M'04) received both the B.Sc. degree in science and the Ph.D. degree in physics from the University College, Cork, Ireland.

In 1998, he joined Corning, Inc., at Corning, New York, as a Senior Development Scientist, working on novel fiber optical amplifiers for telecommunications. Later, he joined Optimight Communications, San Jose, CA, as a Senior Optical Engineer, working on ultralong-haul high-capacity systems. In 2002, he rejoined University College, Cork, as a Senior Researcher. He is currently the Science Foundation Ireland Principal Investigator. His major research interests include semiconductor lasers and high-speed systems where he has made significant contributions in both an academic and industrial setting. 